\title{
ON A GENERALIZED MOMENT PROBLEM. II
}

\author{
J. S. HWANG AND G. D. LIN
}

\begin{abstract}
Recently, we have extended the well-known Müntz-Szász theorem by showing that if $f(z)$ is absolutely continuous and $\left|f^{\prime}(x)\right| \geqslant k>0$ a.e. on $(a, b)$, where $a \geqslant 0$ and if $\left\{n_{p}\right\}$ is a sequence of positive numbers tending to infinity and satisfying $\sum_{p=1}^{x} 1 / n_{p}=\infty$, then the sequence $\left\{f(x)^{n_{p}}\right\}$ is complete on $(a, b)$ if and only if $f(x)$ is strictly monotone on $(a, b)$. We now apply Zarecki's theorem to improve the condition " $\left|f^{\prime}(x)\right| \geqslant k>0$ a.e. on $(a, b)$ " by the condition " $f^{\prime}(x) \neq 0$ a.e. on $(a, b)$ ". Furthermore, we extend some well-known theorems of Picone, Mikusiński, and Boas.
\end{abstract}

1. Introduction. Let $L(a, b)$ be the space of all summable functions defined on the finite interval $(a, b)$. As usual (see R. P. Boas [2, p. 234]) a sequence of functions $f_{n}(x)$ is complete on $(a, b)$ if for any $g \in L(a, b)$, the equalities

$$
\int_{a}^{b} f_{n}(x) g(x) d x=0, \quad n=1,2, \ldots,
$$

imply that $g(x)=0$ a.e. (almost everywhere) on $(a, b)$.

Recently, in [3], we have proved the following extension of the Müntz-Szász theorem (see Boas [2, p. 235]).

THEOREM 1. Let $\left\{n_{p}\right\}$ be a sequence of positive numbers tending to infinity and satisfying

$$
\sum_{p=1}^{\infty} \frac{1}{n_{p}}=\infty .
$$

Let $f(x)$ be a function absolutely continuous on $(a, b)$ with $f(a) f(b) \geqslant 0$, and let its derivative satisfy $\left|f^{\prime}(x)\right| \geqslant k>0$ a.e. on $(a, b)$. Then the sequence $\left\{f(x)^{n_{p}}\right\}$ is complete on $(a, b)$ if and only if the function $f(x)$ is monotone on $(a, b)$.

In this note, we shall improve Theorem 1 as follows.

THEOREM 2. The assertion of Theorem 1 can be extended by replacing the condition " $\left|f^{\prime}(x)\right| \geqslant k>0$ a.e. on $(a, b)$ " by the condition " $f^{\prime}(x) \neq 0$ a.e. on $(a, b)$ ".

2. Inverse function. To prove Theorem 2 , we shall first study the inverse of a monotone function $f(x)$ on $(a, b)$, where monotone function means either strictly increasing or decreasing on $(a, b)$. As usual, we let $f^{-1}(x)$ be the inverse of $f(x)$. We

Received by the editors March 25, 1983.

1980 Mathematics Subject Classification. Primary 30B60; Secondary 26A48.

Key words and phrases. Completeness, moment problem, absolutely continuous and monotone function. 
shall need the following theorem of Zarecki (see Natanson [5, I, p. 271] or Saks [6, p. 128]).

LEMMA 1. Let $f(x)$ be a monotone function on $(a, b)$. Then a necessary and sufficient condition that the inverse $f^{-1}(x)$ be absolutely continuous is that $f^{\prime}(x) \neq 0$ a.e. on $(a, b)$.

We note that the hypothesis " $f^{\prime}(x) \neq 0$ a.e. on $(a, b)$ " in Theorem 2 serves to ensure that the inverse $f^{-1}(x)$ is absolutely continuous on $(a, b)$.

3. Proof of Theorem 2. If $f(x)$ is not monotone on $(a, b)$, then the sequence $\left\{f(x)^{n_{p}}\right\}$ is incomplete on $(a, b)$ due to [3].

Conversely, let $f(x)$ be, say, strictly increasing on $(a, b)$. We shall prove that the sequence $\left\{f(x)^{n_{r}}\right\}$ is complete on $(a, b)$. For this, we consider an arbitrary function $g \in L(a, b)$ such that

$$
\int_{a}^{h} f(x)^{n_{p}} g(x) d x=0, \quad p=1,2, \ldots
$$

Since $f^{\prime}(x) \neq 0$ a.e. on $(a, b)$, it follows from Lemma 1 that the inverse $f^{-1}(x)$ is absolutely continuous on $(f(a), f(b))$. This allows us to change from a Stieltjes to a Lebesgue integral (see [5, I, p. 265 and II, p. 236]). For this, we write $y=f(x)$ and $x=f^{-1}(y)$. Then by (2), we obtain

$$
\int_{f(a)}^{f(b)} y^{n_{p}} g\left(f^{-1}(y)\right)\left(f^{-1}(y)\right)^{\prime} d y=0, \quad p=1,2, \ldots
$$

Since the function $g\left(f^{-1}(y)\right)\left(f^{-1}(y)\right)^{\prime}$ is summable on $(f(a), f(b))$, it follows from (3) and the Müntz-Szász theorem that

$$
g\left(f^{-1}(y)\right)\left(f^{-1}(y)\right)^{\prime}=0 \quad \text { a.e. on }(f(a), f(b)) .
$$

The function $f(x)$ is absolutely continuous on $(a, b)$ so by Lemma 1 , we can see that the derivative of the inverse function $\left(f^{-1}(y)\right)^{\prime} \neq 0$ a.e. on $(f(a), f(b))$. This yields $g(x)=0$ a.e. on $(a, b)$, and therefore the sequence $\left\{f(x)^{n_{p}}\right\}$ is complete on $(a, b)$. This completes the proof.

4. Picone's theorem. Early in the fifties, Boas [1] proved the following extension of Picone's theorem, which also extended to that of Mikusinski [4].

THEOREM B. Let $\left\{n_{p}\right\}$ be a sequence of positive numbers satisfying (1) and $n_{q}-n_{p}>(q-p) d$, where $d>0$ and $p<q$. If $g \in L(a, b)$, where $a \geqslant 0$, and if for some $c$ with $a<c<b$,

$$
\int_{a}^{b} x^{n_{p}} g(x) d x=O\left(c^{n_{p}}\right), \quad p=1,2, \ldots,
$$

then $g(x)=0$ a.e. on $(a, b)$.

In fact, Boas considered a sequence of complex numbers $n_{p}$ with additional assumptions. For simplicity, we consider only the positive real case. We shall extend the above uniqueness theorem of Boas as follows. 
THEOREM 3. Let $f(x)$ be a function absolutely continuous and monotonically increasing on $(a, b)$ whose derivative $f^{\prime}(x) \neq 0$ a.e. on $(a, b)$, where $f(a) \geqslant 0$. Let $\left\{n_{p}\right\}$ be $a$ sequence of positive numbers satisfying (1) and $n_{q}-n_{p}>(q-p) d$, where $d>0$ and $p<q$. If $g \in L(a, b)$ and if for some $c$ with $f(a)<c<f(b)$,

$$
\int_{a}^{b} f(x)^{n_{p}} g(x) d x=O\left(c^{n_{p}}\right), \quad p=1,2, \ldots,
$$

then $g(x)=0$ a.e. on $(a, b)$.

Proof. As before, we let $y=f(x)$ and $x=f^{-1}(y)$. Then equation (4) becomes

$$
\int_{f(a)}^{f(h)} y^{n_{p}} g\left(f^{-1}(y)\right)\left(f^{-1}(y)\right)^{\prime} d y=O\left(c^{n_{p}}\right) .
$$

Since the function $g\left(f^{-1}(y)\right)\left(f^{-1}(y)\right)^{\prime}$ is summable on $(f(a), f(b))$, it follows from Theorem B that

$$
g\left(f^{-1}(y)\right)\left(f^{-1}(y)\right)^{\prime}=0 \quad \text { a.e. on }(f(a), f(b)) .
$$

As before, we know that the function $\left(f^{-1}(y)\right)^{\prime} \neq 0$ a.e. on $(f(a), f(b))$. This yields the assertion $g(x)=0$ a.e. on $(a, b)$.

We shall now consider the following related theorem of Boas [1] about the asymptotic behaviour of moments.

THEOREM $\mathrm{B}^{*}$. If $g(x)$ is a function in $L(a, b)$, where $a \geqslant 0$, and does not vanish almost everywhere in a neighborhood of $b$, then

$$
\lim _{n \rightarrow \infty} \sup \left|\int_{a}^{b} x^{n} g(x) d x\right|^{1 / n}=b .
$$

By the same argument as in Theorem 3 together with Theorem B*, we obtain the following asymptotic behaviour of generalized moments.

THEOREM 4. Let $f(x)$ be a function absolutely continuous and monotonically increasing on $(a, b)$ whose derivative $f^{\prime}(x) \neq 0$ a.e. on $(a, b)$, where $f(a) \geqslant 0$. If $g \in L(a, b)$ and does not vanish a.e. in a neighborhood of $b$, then

$$
\lim _{n \rightarrow \infty} \sup \left|\int_{a}^{b} f(x)^{n} g(x) d x\right|^{1 / n}=f(b) .
$$

The conditions of $f(x)$ in the above theorems could be improved. To discuss this, we shall now prove the following extension of Boas' theorem [2, p. 233].

THEOREM 5. Let $f(x)$ be a nonnegative function monotonically increasing on $(a, b)$ and continuous in a neighborhood of $b$. If $g(x)$ is summable and nonnegative on $(a, b)$, and does not vanish a.e. in a neighborhood of $b$, then

$$
\lim _{n \rightarrow \infty}\left\{\int_{a}^{h} f(x)^{n} g(x) d x\right\}^{1 / n}=f(b) .
$$

Proof. For each sufficiently small $\varepsilon>0$, we have

$$
\begin{aligned}
(f(b-\varepsilon))^{n} \int_{b-\varepsilon}^{b} g(x) d x & \leqslant \int_{b-\varepsilon}^{b} f(x)^{n} g(x) d x \\
& \leqslant \int_{a}^{b} f(x)^{n} g(x) d x \leqslant f(b)^{n} \int_{a}^{b} g(x) d x .
\end{aligned}
$$


By taking the $n$th roots and applying the hypothesis of $g(x)$, we obtain

$$
\begin{aligned}
f(b-\varepsilon) & \leqslant \lim _{n \rightarrow \infty}\left\{\int_{a}^{b} f(x)^{n} g(x) d x\right\}^{1 / n} \\
& \leqslant \varlimsup_{n \rightarrow \infty}\left\{\int_{a}^{b} f(x)^{n} g(x) d x\right\}^{1 / n} \leqslant f(b) .
\end{aligned}
$$

The assertion now follows from the continuity of $f(x)$ in a neighborhood of $b$.

\section{REFERENCES}

1. R. P. Boas, Jr., Remarks on a moment problem, Studia Math. 13 (1953), 59-61.

2. Entire functions, Academic Press, New York, 1954.

3. J. S. Hwang, On a generalized moment problem, Proc. Amer. Math. Soc. 87 (1983), 88-89.

4. J. G. Mikusinski, Remarks on the moment problem and a theorem of Picone, Colloq. Math. 2 (1951), 138-141.

5. I. P. Natanson, Theory of functions of a real variable, Ungar, New York, Vol. I, 1955 and Vol. II, 1960.

6. S. Saks, Theory of the integral, Monografie Matematyczne, PWN, Warsaw, 1937.

Institute of Mathematics, Academia Sinica, Taipei, Taiwan

Institute of Statistics, Academia Sinica, Taipei, Taiwan 